OPEN ACCESS

Edited by:

Baolei Jia

Chung-Ang University, South Korea

Reviewed by:

Sonja-Verena Albers,

Albert Ludwigs University of Freiburg,

Germany

Nils-Kaare Birkeland,

University of Bergen, Norway

*Correspondence:

Yulong Shen

yulgshen@sdu.edu.cn

Specialty section:

This article was submitted to

Extreme Microbiology,

a section of the journa

Frontiers in Microbiology

Received: 12 May 2017

Accepted: 23 October 2017

Published: 07 November 2017

Citation:

Huang Q, Zhong Q, Mayaka JBA,

Ni J and Shen Y (2017)

Autophosphorylation

and Cross-Phosphorylation of Protein

Kinases from the Crenarchaeon

Sulfolobus islandicus.

Front. Microbiol. 8:2173.

doi: 10.3389/fmicb.2017.02173

\section{Autophosphorylation and Cross-Phosphorylation of Protein Kinases from the Crenarchaeon Sulfolobus islandicus}

\author{
Qihong Huang, Qing Zhong, Joseph B. A. Mayaka, Jinfeng Ni and Yulong Shen* \\ State Key Laboratory of Microbial Technology, Shandong University, Jinan, China
}

Protein phosphorylation, one of the most important post-translational modifications, regulates almost every cellular process. Although signal transduction by protein phosphorylation is extensively studied in Eukaryotes and Bacteria, the knowledge of this process in archaea is greatly lagging behind, especially for Ser/Thr/Tyr phosphorylation by eukaryotic-like protein kinases (ePKs). So far, only a few studies on archaeal ePKs have been reported, most of which focused on the phosphorylation activities in vitro, but their physiological functions and interacting network are still largely unknown. In this study, we systematically investigated the autophosphorylation and cross-phosphorylation activities of ePKs from Sulfolobus islandicus REY15A using proteins expressed in Escherichia coli or S. islandicus. In vitro kinase assay showed that 7 out of the 11 putative ePKs have autophosphorylation activity. A protein Ser/Thr phosphatase, SiRe_1009, was able to dephosphorylate various autophosphorylated ePKs, confirming that these proteins are Ser/Thr kinases. Two ePKs, SiRe_2030 and SiRe_2056, homologs of typical eukaryotic PKs involved in peptide synthesis in response to various cellular stresses, exhibit highly efficient phosphorylation activities on both themselves and other ePKs. Overexpression of the protein kinases in vivo revealed that elevated level of either SiRe_1531 or SiRe_2056 inhibited the cell growth of $S$. islandicus cells. Finally, a phosphorylation network of the protein kinases was proposed and their putative physiological roles were discussed.

\footnotetext{
Keywords: archaea, Sulfolobus islandicus, protein phosphorylation, protein kinase, cross-phosphorylation, regulatory network
}

\section{INTRODUCTION}

Protein phosphorylation is a reversible post-translational modification that regulates almost all cellular processes, such as cell cycle progression, cell mobility, DNA replication and repair (Humphrey et al., 2015). Abnormal protein phosphorylation/dephosphorylation in human cells is frequently either a driver or direct consequence of many diseases. Therefore, many protein kinases and phosphor-sites were designed as the targets of medicines (Cohen, 2002). Although protein

Abbreviations: $\mathrm{ABC1}$, activator of bcl complex; ePK, eukaryotic-like protein kinase; eSTK, eukaryotic-like Ser/Thr kinase; HRD, His-Arg-Asp; KD, protein kinase catalytic domain; PTP, protein tyrosine phosphatase; RIO, right open reading frame; TM, transmembrane; TPR, tetratricopeptide repeat; wHTH, winged helix-turn-helix. 
phosphorylation has been extensively investigated in eukarya, the regulation mechanisms are still far from clear due to the presence of large amount of protein kinases/phosphatases (at least 518 protein kinases and 156 protein phosphatases in human) and their complex regulatory networks (Manning et al., 2002; Shi, 2009).

Protein kinases in bacteria were discovered later than those in eukaryotes. A number of previous studies in bacteria focused on histidine kinases in two-component systems, which was considered as the main regulatory phosphorylation in bacteria (Casino et al., 2010). The system contains two components, a sensor histidine kinase and a response regulator. The activated forms of the former would specifically recognize and phosphorylate the latter (Podgornaia and Laub, 2013). This linear signal transfer is different from the network regulatory pattern in eukaryotes. However, genome sequencing and proteomic studies have revealed that there are many eSTK in bacteria (Pereira et al., 2011). Recent studies on the eSTKs from Mycobacterium tuberculosis and the eSTKs and bacterial tyrosine kinases from Bacillus subtilis revealed that bacterial protein kinases exhibited both autophosphorylation and cross-phosphorylation activities on Ser, Thr, and Tyr residues. It seems that bacteria also have a regulatory network and/or cascades of protein kinases as those in eukaryotes. (Baer et al., 2014; Shi et al., 2014).

All sequenced archaeal genomes encode eukaryotic protein Ser/Thr/Tyr kinases and phosphatases, although the numbers are much fewer than those in eukaryotes (Kennelly, 2003), while histidine kinases are mainly found in euryarchaeota (Spudich and Stoeckenius, 1980). Archaeal ePKs contain most conserved subdomains of typical PKs. A number of studies on archaeal ePKs were performed in Crenarchaeota, especially in Sulfolobus (Lower and Kennelly, 2003; Albers and Driessen, 2005; LaRondeLeBlanc et al., 2005; Hecker et al., 2009; Haile and Kennelly, 2011; Ray et al., 2015; Haurat et al., 2017; Hoffmann et al., 2017). In Sulfolobus solfataricus, there are 1318 phosphorylated sites in 540 proteins in total (Esser et al., 2012). These proteins contain most $(21 / 26)$ clusters of orthologous groups, indicating that archaeal protein phosphorylation participates in most biological processes (Makarova et al., 2007). Sulfolobus cells possess at least two protein phosphatases. Deletion of saci_pp2a, one of the two phosphatase resulted in pronounced alterations in growth, cell shape and cell size (Reimann et al., 2013). The expression of the genes encoding components of motility system, the respiratory chain and transcriptional regulators, and their phosphorylation levels significantly changed in the deletion mutants (Reimann et al., 2013). In addition, it was revealed that the SIRV2 virus could not infect the $S$. solfataricus strain with a mutation in an ePK gene, SsoPK2 (Deng et al., 2014), whereas deletion of the SsoPK2 homolog gene in S. acidocaldarius resulted in deficiency of pili formation (Henche et al., 2012). A report on a typical ePK from S. acidocaldarius, Saci1193, showed that the protein stimulated the expression of archaella genes probably by phosphorylating two negative regulatory proteins: ArnA and ArnB (Reimann et al., 2012). Very recently, deletion of either Saci1193 or Saci1181, another ePK gene, resulted in reduced cell motility, while deletion of ePK gene Saci1694 led to hypermotility. Saci1193 and Saci1181 are upregulated during starvation whereas Saci1694 is constitutively expressed. Both Saci1193 and Saci1694 phosphorylated ArnB at its C-terminus (Haurat et al., 2017; Hoffmann et al., 2017). These studies suggested that the ePKs play complex regulatory roles in controlling the expression of archaellum components. The limited functional studies of ePKs mainly focus on the regulation of pili and archaella formation. However, other potential roles of the ePKs and the physiological functions of other archaeal ePKs are largely unknown.

The archaeal ePKs seem to harbor a hierarchy of regulatory networks since they exhibit the characteristics of eSTKs. All typical protein kinases (Hanks-type kinases) contain a conserved catalytic domain folding into two lobes, a smaller amino-terminal lobe (N-lobe) and a larger carboxy-terminal lobe (C-lobe) connected by a hinge region (Hanks and Hunter, 1995). The domain is further divided into 12 subdomains. There are several important residues for catalytic activity: the Lys residue in subdomain II; the conserved Asp in the subdomain VIb, which is likely to be the catalytic base involved in the phosphotransfer reaction, as well as the invariant Asp in subdomain VII, which functions in the orientation and anchoring of the ATP (Hanks and Hunter, 1995; Hanks, 2003). It appears as a common mechanism in eukaryotes that Ser or Thr residues in the activation loop of PKs, a conserved peptide bordered by the subdomains VII and VIII, is phosphorylated to activate PKs' activities (Nolen et al., 2004). It was shown that several Sulfolobus ePKs contained the activation loop, indicative of a kinase crosstalk similar to that in eukaryotes and bacteria (Esser et al., 2016). So, it is interesting to unveil the hierarchy of archaeal ePKs regulatory networks.

In this study, we systematically purified and analyzed eleven putative ePKs from S. islandicus. Their autophosphorylation and cross-phosphorylation activities were investigated. The effects of ePK overexpression on Sulfolobus cell growth were also analyzed. Based on both in vitro and in vivo results, we propose a framework of the phosphorylation network of the protein kinases. Their physiological roles of these protein kinases were also discussed.

\section{MATERIALS AND METHODS}

\section{Strains and Growth Conditions}

Sulfolobus islandicus strain E233S ( $\triangle$ pyrEF $\triangle$ lacS, Table 1) (hereafter E233S) was grown at $75^{\circ} \mathrm{C}$ in the mineral salt medium supplemented with $0.2 \%$ (wt/vol) sucrose (S), 0.2\% (wt/vol) tryptone $(\mathrm{T})$, a mixed vitamin solution $(\mathrm{V})$, and $0.01 \%(\mathrm{wt} / \mathrm{vol})$ uracil (U) (named MTSVU medium), as described previously (Deng et al., 2009). MSV medium supplemented with $0.2 \%$ casamino acid (C) was used for cultivating uracil prototrophic strains. MTV medium supplemented with $0.2 \%$ arabinose (A) was used for protein expression. Phytagel $(0.8 \%$ [wt/vol]) was added in the medium for making plates.

\section{Plasmids Construction}

To construct the plasmids for expressing N-His-tagged protein kinases or protein phosphatases in Escherichia coli, each gene 
TABLE 1 | Sulfolobus strains used in this study.

\begin{tabular}{lll}
\hline Strains & Genotype & Source \\
\hline Sulfolobus islandicus REY15A (E233S) & spyrEF $\Delta$ lacS & Deng et al., 2009. \\
E233S/pSeSD-0101KD-C-His, & E233S with & This work \\
E233S/pSeSD-0171-C-His, & various protein & \\
E233S/pSeSD-0181-C-His, & kinase genes & \\
E233S/pSeSD-1057-C-His, & on the & \\
E233S/pSeSD-1531-C-His, & expression & \\
E233S/pSeSD-1570-C-His, & vector pSeSD & \\
E233S/pSeSD-1639-C-His, & harboring araS & \\
E233S/pSeSD-1810-C-His, & promoter & \\
E233S/pSeSD-2030-C-His, & coding for & \\
E233S/pSeSD-2056KD-C-His, & C-His-tagged & \\
E233S/pSeSD-2600-C-His & protein kinases & \\
\hline
\end{tabular}

(or a gene fragment containing kinase domain) was amplified by PCR using their corresponding primers (NdeI-F/SalI-R) listed in Supplementary Table S1. The NdeI restriction sites in SiRe_0181, SiRe_2056, SiRe_2600, and SiRe_0241 genes were mutated using splicing by overlap extension (SOE) PCR. The PCR product of each gene was digested and ligated into the NdeI and SalI sites of the pET15b vector. For expression of inactive protein kinases in E. coli, the conserved Asp or Glu within the core catalytic motif of each gene was mutated by SOE PCR using the wild type gene as template and inserted into pET15b.

The vectors for overexpression of C-His-tagged protein kinases in S. islandicus were constructed by amplification of each gene (or kinase domain) using their corresponding primers (NdeI-F/Nostop-SalI-R) and insertion of the gene fragment into the NdeI and SalI sites of the vector pSeSD (Supplementary Table S2) carrying the pyrEF marker (Peng et al., 2012), yielding kinase overexpression vector. The primers used for PCR are listed in Supplementary Table S1.

\section{Transformation of S. islandicus Strains and Determination of the Growth}

The expression plasmids were transformed into $S$. islandicus cells by electroperation as previously described (Deng et al., 2009). To obtain growth curves, cells were grown to early log-phase and transferred for 3-4 times before the measurement. The initial $\mathrm{OD}_{600}$ value was $0.03-0.04$ and the ODs were measured every 6 or $12 \mathrm{~h}$. A growth curve was made based on the data from at least three parallel experiments.

\section{Western Blot Analysis}

Two milliliters of the cultures in early log-phase was collected by spinning down. The cells were resuspended in $40 \mu \mathrm{l}$ of a buffer containing $50 \mathrm{mM}$ Tris- $\mathrm{HCl} \mathrm{pH} 8.0,100 \mathrm{mM}$ $\mathrm{NaCl}$ and $10 \mu \mathrm{l}$ of $5 \times$ SDS-PAGE loading buffer. The mixture was boiled for $10 \mathrm{~min}$ and loaded onto a SDS-PAGE gel. The proteins in the PAGE gel were transferred onto a PDVF membrane. The membrane was incubated with anti- $6 \times$ His antibodies and HRP-labeled goat anti-mouse IgG under standard Western blot conditions. The image was obtained by ImageQuant 400 (GE Healthcare, Buckinghamshire, United Kingdom).

\section{Protein Purification}

To purify wild type and mutant protein kinases from E. coli, the protein expression was induced at either $37^{\circ} \mathrm{C}$ for $4 \mathrm{~h}$ or $16^{\circ} \mathrm{C}$ for $12-16 \mathrm{~h}$. The cell pellets were resuspended in buffer A (50 mM Tris pH 8.0 or $9.0,200 \mathrm{mM} \mathrm{NaCl}$, and $5 \%$ glycerol) and lysed by sonication. The soluble proteins were heated at $70^{\circ} \mathrm{C}$ for $30 \mathrm{~min}$ and the supernatants after centrifugation were loaded onto a Ni-NTA column pre-equibilirated with buffer A. Unbound proteins were washed by wash buffer (buffer A supplemented with $40 \mathrm{mM}$ imidazole) and target proteins were eluted by elute buffer (buffer A supplemented with $250 \mathrm{mM}$ imidazole). The eluted fractions containing target proteins were pooled and concentrated. The proteins were subsequently purified by gel filtration using a Superdex ${ }^{\mathrm{TM}} 200$ 10/300 column (GE Health, United Kingdom) in the corresponding buffer. Fractions containing the purified proteins were collected, aliquoted, and stored at $-80^{\circ} \mathrm{C}$ after frozen with liquid nitrogen. The protein concentration was determined by the Bradford method with bovine serum albumin as the standard. The procedure for purification of SiRe_0101KD-C-His from S. islandicus was the same as above except that the protein was induced by arabinose at $75^{\circ} \mathrm{C}$ for $12 \mathrm{~h}$ and purified without heat-treatment. The fractions containing SiRe_0101KD-C-His were analyzed by SDS-PAGE and the whole lane was cut for mass spectrometry analysis by BGI (Beijing Protein Research Center Co., Ltd.).

\section{In Vitro Kinase Assay}

To analyze the autophosphorylation activities of protein kinases, a certain mount ( 1 or $2 \mu \mathrm{M}$ as specified) of wild type protein kinase was added into a reaction mixture $(20 \mu \mathrm{l})$ containing 25 mM Tris- $\mathrm{HCl}$ pH 8.0, $50 \mathrm{mM} \mathrm{NaCl}, 5 \mathrm{mM} \mathrm{MgCl} 2$ (or $\mathrm{MnCl}_{2}$ ), $2 \mathrm{mM}$ DTT, $4.2 \mathrm{nM}\left[\gamma_{-}{ }^{32} \mathrm{P}\right]$ ATP (111 TBq/mmol, PerkinElmer), and $50 \mu \mathrm{M}$ cold carrier ATP. The mixture was incubated at $65^{\circ} \mathrm{C}$ for $30 \mathrm{~min}$ and the reaction was stopped by adding $5 \times$ SDSPAGE loading buffer and boiling for $10 \mathrm{~min}$. The samples were analyzed by $12 \%$ or $15 \%$ SDS-PAGE. The autoradiographs were quantified by the software ImageQuant 5.2.

For detecting the cross-phosphorylation activities between two protein kinases, the reaction is the same as above except that an inactive protein kinase $(2 \mu \mathrm{M})$ was added as the substrate. The dephosphorylation activities of the protein phosphatases were analyzed by adding a protein phosphatase $(2 \mu \mathrm{M})$ in the autophosphorylation reaction above.

\section{RESULTS}

\section{Bioinformatics Analysis and Purification of $S$. islandicus Eukaryotic-Like Protein Kinases}

There are ten potential ePKs encoded in the $S$. solfataricus genome (Kennelly, 2014). As S. islandicus has highly close phylogenetic relationship with $S$. solfataricus, the homologs of these 10 ePKs (SiRe_0101, SiRe_0171, SiRe_0181, SiRe_1057, SiRe_1531, SiRe_1570, SiRe_1810, SiRe_2030, SiRe_2056, and SiRe_2600) were all found in S. islandicus REY15A by 
TABLE 2 | Sulfolobus islandicus protein kinase homologs in other Sulfolobus species.

\begin{tabular}{lccc}
\hline S. islandicus & S. solfataricus & S. acidocaldarius & S. tokodaii \\
\hline SiRe_0101 & Sso2291 & - & - \\
SiRe_0171 & Sso2374 & Saci_0965 & STK_05130 \\
SiRe_0181 & Sso2387 & Saci_2317 & STK_05220 \\
SiRe_1057 & Sso1038 & Saci_1289 & STK_09530 \\
SiRe_1531 & Sso0469 & Saci_0435 & STK_01810 \\
SiRe_1570 & Sso0433 & Saci_0850 & STK_03640 \\
SiRe_1639 & Sso0361 & Saci_1477 & STK_13820 \\
SiRe_1810 & Sso0197 & Saci_0796 & STK_02330 \\
SiRe_2030 & Sso3207 & Saci_1193 & STK_08100 \\
SiRe_2056 & Sso3182 & - & - \\
SiRe_2600 & Sso2605 & Saci_1664 & STK_16520 \\
\hline
\end{tabular}

BLAST analysis (Table 2). In addition, SiRe_1639 is also annotated as a $\mathrm{Mn}^{2+}$-dependent serine/threonine protein kinase containing a protein kinase catalytic domain (KD) according to the genomic information of S. islandicus REY15A (Figure 1A) (Guo et al., 2011). All these 11 ePKs contain a putative conserved Asp residue (or Glu for SiRe_1057, see below) at the HRD motif within the catalytic domain (subdomain VIb) as revealed by sequence alignment (Figure 1B) (Hanks and Hunter, 1995). The Asp sites in SiRe_0181 and SiRe_1531 were identified previously by sequence analysis (Sso2387 and Sso0469 in S. solfataricus, respectively). Several ePKs also harbor other domains that may facilitate their functions in the cell, such as TM domain, wHTH, and TPR (Figure 1A).

To investigate the phosphorylation network of these ePKs, the proteins were expressed and purified from E. coli. Initially, all ePK proteins were expressed but the expressed proteins were mostly, or at least partially, insoluble during cell lysis by sonication (data not shown). After optimizing the conditions for protein induction and purification, we were able to get the full length of the majority of these proteins except for SiRe_0101 and SiRe_2056 (Figure 2A). Since both ePKs contain a TM domain at their N-terminal which would probably affect

A

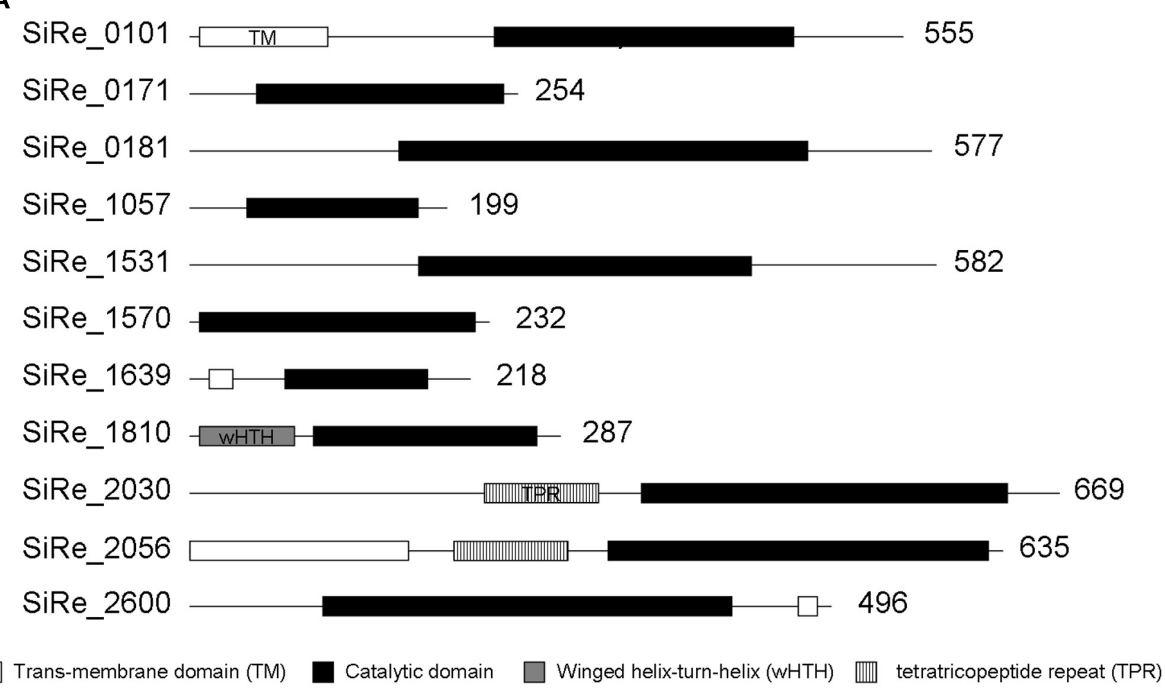

B

\begin{tabular}{|c|c|c|c|c|}
\hline SiRe_0181 & 480 & RRVSS--WDF & --AGLSIDDV-----KREMERRALFL & 527 \\
\hline SiRe_1531 & 320 & ISQKPLLYTFISKIDRF-MRERKLTVDILII & DSIRRLLDEIERLGKDAHLKE--HSSLFT & 376 \\
\hline SiRe_1810 & 201 & -LRLDEILEKILQTMRIAYTICRITHGDISP & -YNVLIDK---------HGRPY- & \\
\hline SiRe_0171 & 162 & -EITQELYDDLIQQVEIMTKRAKLVYHFDILEE & --YRVNVY---------------DHKCY- & 202 \\
\hline SiRe_1639 & 91 & DEIREERIEIVAKGLRE-IHEVGYVLGDTKI & --SHFVIVRRSKLAVIDAEQSFKSHRLYY & 147 \\
\hline SiRe_1570 & 108 & VQPLSKIGERIGELTGK-LHSIGIAHGDLTT & --MRLILSSI-----------KRDIF- & 150 \\
\hline SiRe_1057 & 109 & KIERRETIIDLLLRARI-LEEKLIEFKKILIS & PWKNVIVS---------------YNRTY- & 151 \\
\hline iRe_2600 & 236 & -VDNRILAYRVFRLFMYMLLNKDYFHADDFHF & -GRIAVDE--- & 277 \\
\hline SiRe_0101 & 367 & YRIVGILIRDVAKALDI-IHSSGYVHLDVKFF & --QNIYFSKRPGSKEKEILSNLTSGKWV & 423 \\
\hline SiRe_2030 & 472 & PQVVAFVFSKLAKAVIE-VHKKEGYTPKCDDKF & --SNILFHKKLPRYGEDALNSLLUFEVVP & 528 \\
\hline \multirow[t]{3}{*}{ SiRe_2056 } & 450 & RKIVFIATARIAEALET-IHSEGYWHCDJKP & --QRILFNEKLPPNARLAYDKLKAGGKIIA & 506 \\
\hline & & h hrD k & $\mathrm{N}$ & \\
\hline & & & & \\
\hline
\end{tabular}

FIGURE 1 | Putative eukaryotic-like protein kinases (ePKs) in Sulfolobus islandicus REY15A. (A) Domain organization of the protein kinases. The domains were predicted according to database of NCBI (https://www.ncbi.nlm.nih.gov/) and UniProt (http://www.uniprot.org/) and are shown in boxes with the amino acid numbers being indicated on the right. (B) Alignment of the core catalytic domains of the predicted ePKs. The sequences were analyzed by ClustalW. The conserved HRD motif within subdomain VIb is indicated in dashed boxes. The HRD motif in SiRe_0181 and SiRe_1531 are identified according to the studies on their homologs (Sso2387 and Sso0469, respectively) from S. solfataricus (Lower and Kennelly, 2003; Lower et al., 2004). The consensus sequences of the motifs are depicted above the lines: uppercase, universally conserved amino acid residues; lowercase, highly conserved amino acid residues. 

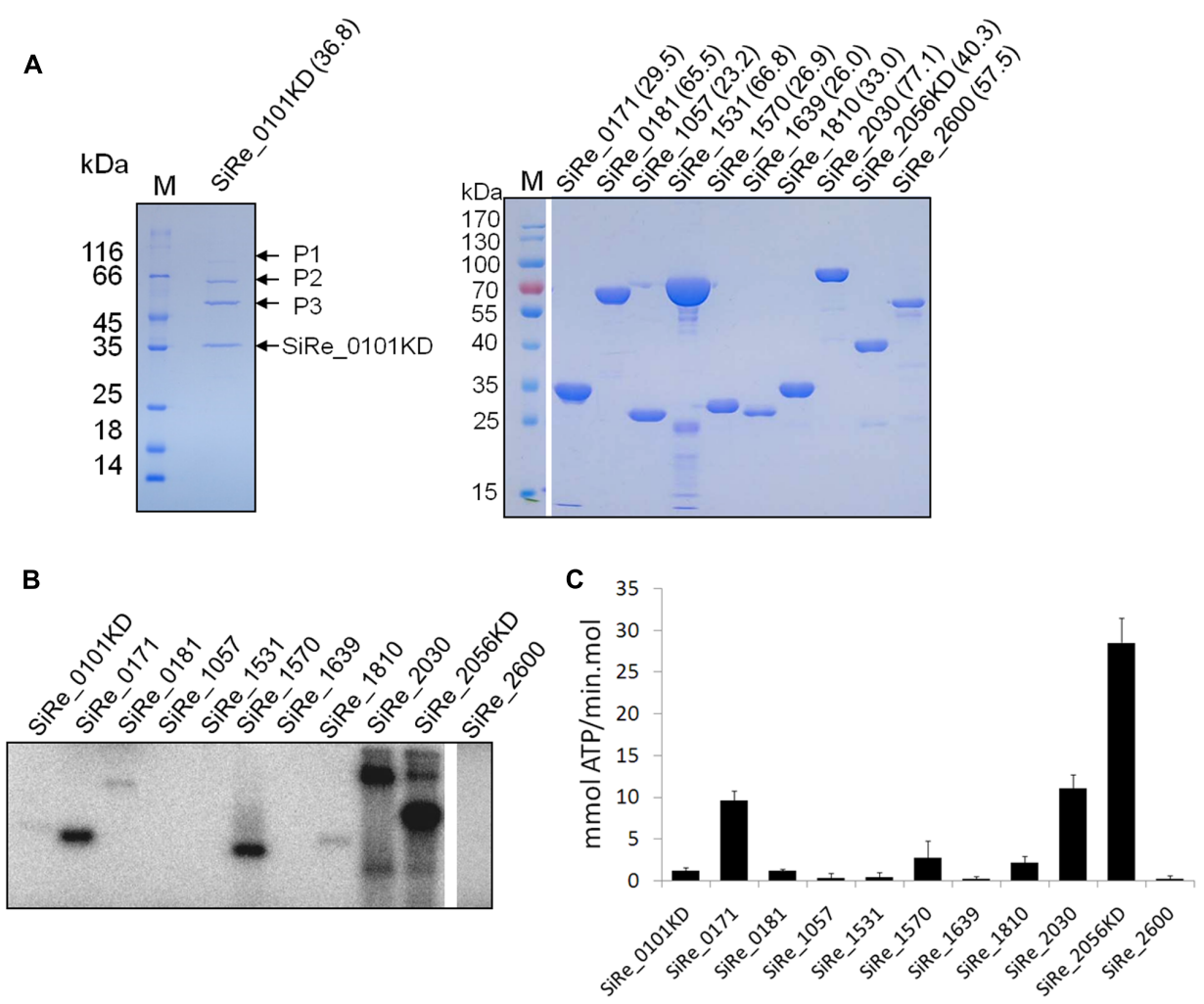

FIGURE 2 | Purification of the eukaryotic-like protein kinases (ePKs) of S. islandicus REY15A and analysis of their autophosphorylation activities. (A) Purification of His-tagged ePKs expressed in Escherichia coli (right) or S. islandicus REY15A (left). The predicted molecular weight (kDa) are shown in the parentheses. M, molecular size marker. P1, P2, and P3, proteins co-purified with SiRe_0101KD. (B) Autophosphorylation activities of S. islandicus ePKs. Each ePK (2 $\mu$ M) was incubated in the reaction mixture containing $4.2 \mathrm{nM}\left[\gamma-{ }^{32} \mathrm{P}\right] \mathrm{ATP}$ and $50 \mu \mathrm{M}$ cold carrier ATP (see Materials and Methods) at $65^{\circ} \mathrm{C}$. The samples were analyzed by $15 \%$ SDS-PAGE. The experiments were performed for at least three times for each enzyme. Representative images of ePKs autophosphorylation activities are shown. (C) The autoradiographs in B are quantified by ImageQuant. The data were obtained from three independent experiments. The bars indicate standard deviation.

their expression in E. coli, we then attempted to purify the catalytic domains (KDs) of both ePKs. However, only soluble SiRe_2056KD was obtained using E. coli expression system. The expression of SiRe_0101 was further tried and the protein was successfully expressed in S. islandicus using shutter vector pSeSD (Peng et al., 2012). Western blot analysis showed that the band of SiRe_0101KD, but not the full length, was visible after purification by Ni-NTA column (Supplementary Figure S1). There were also several other proteins in the fractions $(9-11 \mathrm{ml})$ containing SiRe_0101KD even after purification by gel filtration (Figure 2A).

\section{Autophosphorylation Activities of Various S. islandicus ePKs and Their Dephosphorylation by Two Protein Phosphatases}

Autophosphorylation is the dominant mode of ePK activation and a mechanism of efficient signal amplification (Nolen et al., 2004). It was shown that several S. solfataricus and S. acidocaldarius ePKs exhibited autophosphorylation activities mainly on Ser or Thr (Lower and Kennelly, 2003; Albers and Driessen, 2005; LaRonde-LeBlanc et al.,
2005; Hecker et al., 2009; Haile and Kennelly, 2011; Ray et al., 2015; Haurat et al., 2017; Hoffmann et al., 2017). To systematically analyze the autophosphorylation activities of $S$. islandicus ePKs, each ePK was incubated with $[\gamma-$ $\left.{ }^{32} \mathrm{P}\right] \mathrm{ATP}$ in a reaction mixture for the kinase assay. The results revealed that, among these ePKs, seven ePKs (SiRe_0101KD, SiRe_0171, SiRe_0181, SiRe_1570, SiRe_1810, SiRe_2030, and SiRe_2056KD) exhibited autophosphorylation activities under our experimental conditions with SiRe_2056KD having the highest activity (Figures 2B,C). In addition, SiRe_2030 exhibited higher autophosphorylation activity in the presence of $\mathrm{Mn}^{2+}$ than $\mathrm{Mg}^{2+}$, while the activity of $\mathrm{SiRe}_{-} 1570$ was very low in the presence of $\mathrm{Mg}^{2+}$ (Supplementary Figure S2). Hereafter, $\mathrm{MnCl}_{2}$ was added into the kinase assay mixture for SiRe_1570 and SiRe_2030 in subsequent analysis. The quantitative result showed that SiRe_2056KD was able to incorporate $28 \mathrm{mmol}$ phosphate/min. $\mathrm{mol}$ into itself, which is almost the same as its homolog in S. solfataricus (Ray et al., 2015).

To confirm that the autophosphorylated residues were Ser/Thr or Tyr, two protein phosphatases (SiRe_0241 and SiRe_1009) were purified from E. coli and applied in the dephosphorylation assay (Supplementary Figure S3). SiRe_0241 
belongs to the family of PTP, while SiRe_1009 is a phosphorSer/Thr phosphatase (PP2A, a subfamily of PPP). It was shown that Saci-PTP displayed a phosphatase activity toward both pTyr and pSer/pThr with a much higher activity on pTyr (30- and 131-fold higher as compared with those on pSer and pThr, respectively), but Sso-PTP exhibited phosphohydrolase activity only toward pTyr (Chu and Wang, 2007; Reimann et al., 2013). A phylogenetic analysis on PPPs from three domains of life showed that archaeal PPPs were inherited from the last universal common ancestor (Kennelly, 2014). So far, the studies on archaeal PPPs, including Saci-PP2A, revealed that they all exhibited specific pSer/pThr activity (Solow et al., 1997; Mai et al., 1998; Reimann et al., 2013). Because high amino sequence conservation between SiRe_0241 and Sso-PTP as well as SiRe_1009 and Saci-PP2A, we assume that SiRe_0241 and SiRe_1009 are PTP and PP2A in S. islandicus, respectively. In our dephosphorylation assay (Figure 3), SiRe_0241 (Sis-PTP) only slightly reduced the signal strength, whereas the bands corresponding to all the autophosphorylated proteins were nearly invisible or significantly decreased in the presence of SiRe_1009 (Sis-PP2A). Collectively, our results suggested that most $\mathrm{ePKs}$ phosphorylated themselves on Ser/Thr sites. The results are consistent with those of the phosphoproteomics analysis of the SiRe_0171 and SiRe_2030 homologs in S. acidocaldarius showing that both ePKs were phosphorylated mainly on Ser/Thr sites in vivo (Reimann et al., 2013).

\section{Cross-Phosphorylation Relationship among ePKs}

The complexity of eukaryotic regulation by protein phosphorylation is due to their complicated crossphosphorylation network of protein kinases and signaling cascade (Breitkreutz et al., 2010). Recent studies in M. tuberculosis and Bacillus subtilis indicated that the behavior of bacterial ePKs network was also similar to that in eukaryotes (Baer et al., 2014; Shi et al., 2014). To understand the cross-talk between various ePKs from $S$. islandicus, inactive ePKs were generated by introducing a mutation (Asn or Ala) at the conserved Asp or Glu site (for SiRe_1057) within the catalytic domain of wild type ePKs (Figure 1B and Supplementary Figure S4). Glu residue in SiRe_1057 is highly conserved in all analyzed archaeal homologs (Supplementary Figure S4). As shown in Figure 4A, all site-directed mutants except for SiRe_0181D490N did not have autophosphorylation activity. The Asp within the HRD motif of SiRe_0181 was identified according to the studies on the $S$. solfataricus homologs (Sso2387) which is annotated as a secretion ATPase (Lower and Kennelly, 2003; Albers and Driessen, 2005). However, after substitution of Asp by Asn, the mutant protein still exhibited autophosphorylation activity (Supplementary Figure S5). It seems that the mechanism of SiRe_0181 autophosphorylation was different from other PKs and the conserved Walker A and Walker B motifs may be essential for the activity. To test this, we constructed another SiRe_0181 mutant by replacing Lys in Walker A motif with Ala (SiRe_0181K324A) and examined its kinase activity. The result showed that SiRe_0181K324A did not phosphorylate itself (Supplementary Figure S5). This mutant was used for the subsequent cross-phosphorylation assay.

The cross-phosphorylation activities among various pairs of ePKs were then analyzed using complete set of active and inactive ePKs. We found that SiRe_2030 and SiRe_2056KD were the only two ePKs having highly efficient phosphorylation activity on heterologous ePKs. SiRe_2056KD was able to phosphorylate all other ten ePKs, whereas SiRe_2030 phosphorylated most ePKs except for SiRe_1531 and SiRe_2600 (Figures 4B,C). Interestingly, SiRe_0101KD only phosphorylated SiRe_2056KD, while SiRe_1570 had low activity on SiRe_1057. Other ePKs did not phosphorylate heterologous ePKs regardless of whether they had autophosphorylation activities or not. Taken together, SiRe_2030 and SiRe_2056 may serve as the active ePKs that respond to different cellular stresses in vivo, while the others (excluding SiRe_0101) might be the substrate ePKs.

\section{Effect of ePKs Overexpression on the Growth of S. islandicus}

The analysis of gene overexpression phenotypes provides a unique way to study gene functions, because it can lead to hyper-effects on cells often due to mis-regulation and those strictly regulated in the cell could not be overexpressed (Stevenson et al., 2001; Boyer et al., 2004). To analyze the importance of ePKs in S. islandicus, overexpression strains of each ePK were constructed with pSeSD-based vectors and the C-terminal his-tagged proteins were induced by adding arabinose into the medium. It is confirmed that the pSeSD-based plasmids carrying each kinase gene was maintained in the Sulfolobus cell by plasmid re-extraction and digestion (data not shown). Seven ePKs or their KDs (SiRe_0101KD, SiRe_0181, SiRe_1057, SiRe_1639, SiRe_2600, SiRe_1531, and SiRe_2056) were overexpressed as detected by Western blot analysis against the His-tag, while the expression of SiRe_0171, SiRe_1570, SiRe_1810, and SiRe_2030 were not detectable (Figure 5A). However, only strains overexpressing SiRe_1531 and SiRe_2056 overexpression showed growth retardation (Figures $\mathbf{5 B}, \mathbf{C}$ ). The other strains showed no difference in growth from that of the control carrying empty vector (Figures 5C-E). The strains with pSeSD carrying SiRe_0171, SiRe_1570, SiRe_1810, or SiRe_2030 genes grew normally probably because these ePKs were not overexpressed. The protein levels may be repressed by either transcriptional regulation or protein degradation. The reasons why these kinases could not be overexpressed need further investigation. In addition, expression of SiRe_2056KD also resulted in cell growth retardance.

\section{Mass Spectrometry Analysis of Proteins Co-purified with SiRe_0101KD from S. islandicus}

As we mentioned above, in the fractions of SiRe_0101KD purified from $S$. islandicus by gel filtration following Ni-NTA column purification, several other protein bands were clearly visible on the SDS-PAGE gel. To identify these proteins and provide hints of potential functions of SiRe_0101, the whole lane was cut and 
the protein identity was analyzed by mass spectrometry. More than 100 proteins were identified (Supplementary Table S3). Among those with high score, there were a number of proteins putatively involved in protein folding and degradation, including

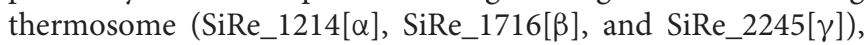
AAA family ATPase of CDC48 subfamily (SiRe_1582 and SiRe_1832), the subunits of proteasome endopeptidase complex

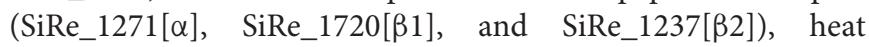
shock protein Hsp20 (SiRe_0216), and prefoldin subunits $\left(\operatorname{SiRe} \_1279[\alpha]\right.$ and $\left.\operatorname{SiRe} \_1653[\beta]\right)$. According to the protein masses and scores, the three visible bands P1, P2, and P3 might be Cdc48 (SiRe_1582, $85.5 \mathrm{kDa}$ ), thermosome (SiRe_1214 or SiRe_1716, $59.7 \mathrm{kDa}$ ), and TIP49 (SiRe_0238, 50.1 kDa), respectively (Figure 2A). Two ePKs, SiRe_0181 and SiRe_1639, were also found in the fraction, as well as other proteins involved in DNA metabolism, translation, and energy production, amino acid transport and metabolism. The relationship of SiRe_0101 with Cdc48, the thermosome, and TIP49 needs further investigation.

\section{DISCUSSION}

Studies on archaeal protein kinases are limited and the majority mainly focused on their in vitro biochemical activities. For the first time, we systematically purified and analyzed the autophosphorylation and cross-phosphorylation activities of eleven putative S. islandicus ePKs. We found that SiRe_0101KD, SiRe_0171, SiRe_0181, SiRe_1570, SiRe_1810, SiRe_2030, and SiRe_2056KD had autophosphorylation activities and SiRe_2030 and SiRe_2056KD exhibited higher activities either on themselves or on most heterologous ePKs than the others. Most eukaryotic PKs which are referred to as RD kinases contain a conserved Arg located adjacent to the key catalytic residue Asp (Krupa et al., 2004). This positively charged Arg inhibits catalysis by the neighboring negatively charged Asp. The inhibition can be removed by phosphorylation of the activation loop which produces negatively charged phosphoamino acids and neutralize the positively charged Arg, resulting in kinase activation (Johnson et al., 1996). Strikingly, all the $S$. islandicus ePKs seem to be non-RD kinases due to lack of the Arg residue and may have different activation mechanisms from that for RD kinases (Figure 1B). It has been shown that some non-RD kinases do not autophosphorylate the activation loop and are either constitutively active or regulated by alternative mechanisms (Dardick et al., 2012). Consistently, several in vitro studies revealed that the phosphorylated sites of archaeal ePKs were not located within the activation loop (LaRonde-LeBlanc et al., 2005; Reimann et al., 2013; Ray et al., 2015).

The three ePKs, SiRe_0101, SiRe_2030, and SiRe_2056, are typical eukaryotic protein kinases, especially those phosphorylating eIF $2 \alpha$ in eukaryotes. Eukaryotic eIF $2 \alpha$ protein kinases phosphorylate eIF2 $\alpha$ to inhibit global polypeptide synthesis in response to a variety of cellular stresses (Donnelly et al., 2013). In vitro kinase assay revealed that S. solfataricus homolog of SiRe_2056, Sso3182, was able to phosphorylate the archaeal homolog of $\operatorname{eIF} 2 \alpha(\operatorname{aIF} 2 \alpha)$, but not on the conserved
Ser51 of eIF2 $\alpha$ (Ray et al., 2015). It was shown that the SiRe_2030 homolog in S. acidocaldarius, Saci_1193, may stimulate archaella expression by phosphorylating two repressors of archaella expression, ArnA and ArnB (Reimann et al., 2012). Saci1193 was up-regulated during starvation and its deletion resulted in reduced cell motility (Hoffmann et al., 2017). In addition, Saci_1193 was up-regulated during G1/S phase transition (Lundgren and Bernander, 2007), while the homolog in S. solfataricus, Sso3207, was transcriptionally repressed after UV radiation (Frols et al., 2007). In our study, SiRe_2056, but not SiRe_2030, could be overexpressed in S. islandicus and resulted in growth retardance. It seems that both SiRe_2030 and SiRe_2056 exhibited efficient phosphorylation ability in vitro and may work as master kinases. Consistently, both ePKs contain a TPR which involves in a variety of protein-protein interactions (Groves and Barford, 1999). However, they may work in different signaling pathways. We speculated that SiRe_2030 might be involved in cell cycle regulation or the responses to harsh conditions such as DNA damage for cell survival so that it could not be overexpressed, while SiRe_2056 overexpression was inducible in the presence of certain cellular stresses and a large amount of the protein with high activity would severely inhibit the cell growth. However, it should be noted that although SiRe_2056KD efficiently phosphorylated all other ePKs in vitro, the membrane localization would allow limited number of the substrate kinases to be accessible in vivo. Due to complex regulatory mechanisms under cellular conditions, the signaling pathways they transmitted may be not as same as the results based on our in vitro assays and need further physiological data to support. Our MS analysis of co-purified proteins of SiRe_0101KD identified many proteins that might participate in protein folding and degradation. In our previous report, only thermosomes were co-purified with a homologous recombination protein HerA using the same protocol (Huang et al., 2015). It seems that the identified proteins with high scores may be those having potential interactions with $\mathrm{SiRe}$ _0101 rather than unspecific binding proteins. However, the proteins with low scores in MS analysis may be contamination due to partial degraded proteins in the gel. Even though the phosphorylation activity of SiRe_0101KD on other co-purified proteins was not detectable in the autophosphorylation assay (Figures 2B, 3A), we could not exclude that they had already been phosphorylated after purification together with SiRe_0101KD from the host cell. It has been revealed that a $S$. acidocaldarius thermosome (SiRe_1214 homolog) and the three subunits of proteasome complex, as well as the proteasome subunits in Haloferax volcanii, can be phosphorylated in vivo, suggesting that protein folding and/or degradation were regulated by protein phosphorylation in which SiRe_0101 may be involved (Humbard et al., 2010; Reimann et al., 2013).

SiRe_1570 belongs to an ancient family of protein kinases, the piD261/Bud32 protein kinase. Studies on the homolog protein from S. solfataricus in vitro showed that it phosphorylated itself and some acid proteins on Ser/Thr, and its activity was stimulated by ADP-ribose (Haile and Kennelly, 2011). A protein complex KEOPS (Kinase Endopeptidase and Other Proteins of Small size), formed by Bud32, Kael (kinase-associated 


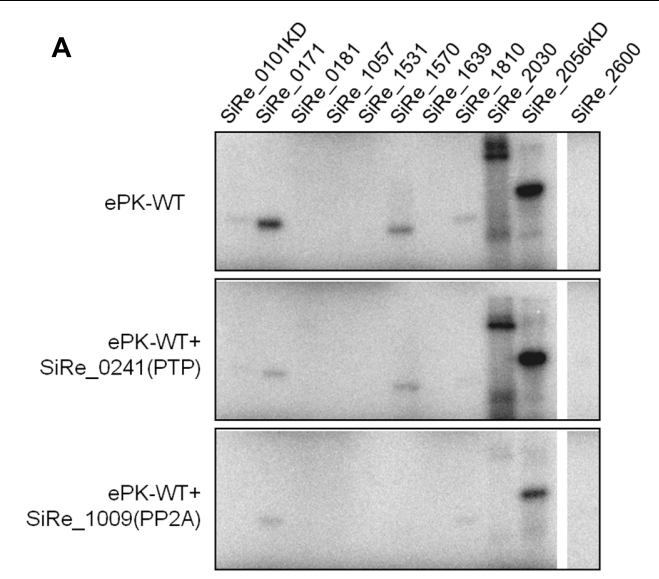

\section{B}

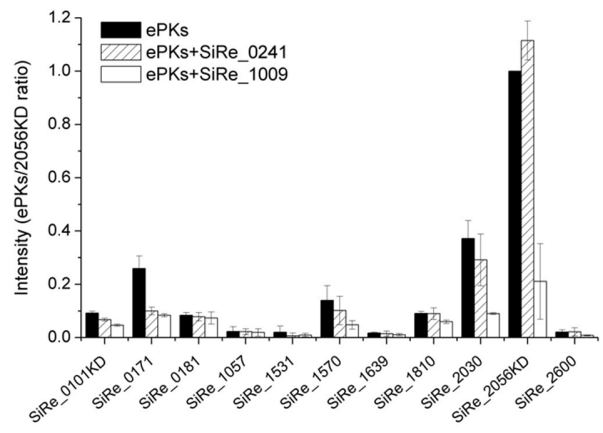

FIGURE 3 | Dephosphorylation of the autophosphorylated S. islandicus ePKs by two protein phosphatases (PPs), SiRe_0241 (protein tyrosine phosphatase, PTP) and SiRe_1009 (phospho-Ser/Thr phosphatase, PP2A). (A) Representative profiles of the phosphorylation (upper panel) and dephosphorylation reactions (middle and lower panels). For the dephosphorylation reactions, each ePK $(1 \mu \mathrm{M})$ was incubated with one of the two PPs in a reaction at $65^{\circ} \mathrm{C}$ and the samples were analyzed by $12 \%$ SDS-PAGE. The experiments were performed for at least three times. Representative images of ePKs autophosphorylation (upper panel), dephosphorylation by SiRe_0241 (middle panel) and SiRe_1009 (lower panel) are shown. (B) Quantitative results of the autoradiographs in (A). The signals of each ePK are normalized to the autophosphorylation signal of SiRe_2056KD. The bars indicate standard deviation.

A

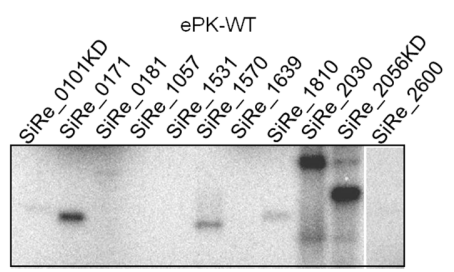

B

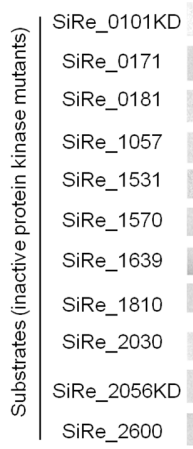

Active protein kinases

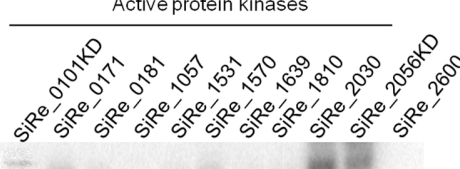

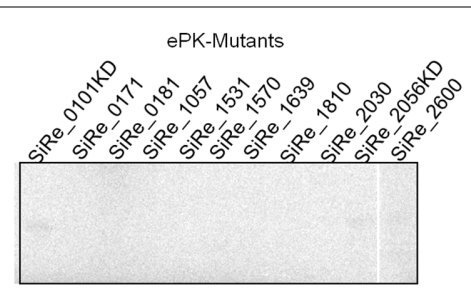

C

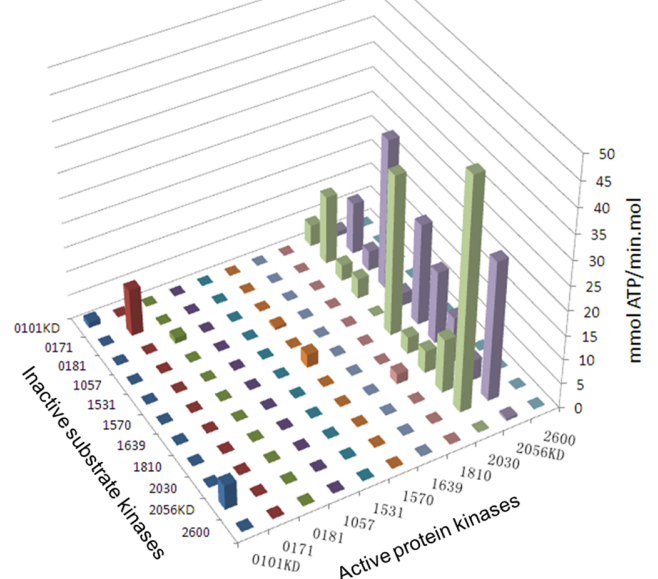

FIGURE 4 | Cross-phosphorylation activities among various ePKs. (A) Autophosphorylation activities of the wild type (WT, left panel) and mutants (M, right panel) ePKs. (B) Cross-phosphorylation activities of each ePK on eleven inactive ePK mutants. Each ePK ( $2 \mu \mathrm{M})$ was incubated with the same concentration of each of the eleven inactive ePKs in the reaction containing $4.2 \mathrm{nM}\left[\gamma-{ }^{32} \mathrm{P}\right] \mathrm{ATP}$ and $50 \mu \mathrm{M}$ cold carrier ATP. The signals on the diagonal indicate autophosphorylation. Off-diagonal bands in each column reflect cross-phosphorylation. The arrow indicates the autophosphorylation signal of wild type SiRe_0171 whose size ( $30 \mathrm{kDa}$ ) is a bit smaller than that of SiRe_1810 (33 kDa). The experiments were performed at least for three times. Representative gel images are shown. (C) Quantitative analysis of the auto/cross-phosphorylation activities in (B). The data were obtained from three independent experiments.

endopeptidase 1) and two small proteins, Cgi121 and Pcc1, are highly conserved throughout archaea and eukaryotes (Hecker et al., 2009). The complex is involved in the biosynthesis of the universal $\mathrm{N}^{6}$-threonylcarbamoyladenosine (t6A) tRNA modification (Srinivasan et al., 2011), regulation of transcription and maintenance of telomere integrity (Downey et al., 2006; 

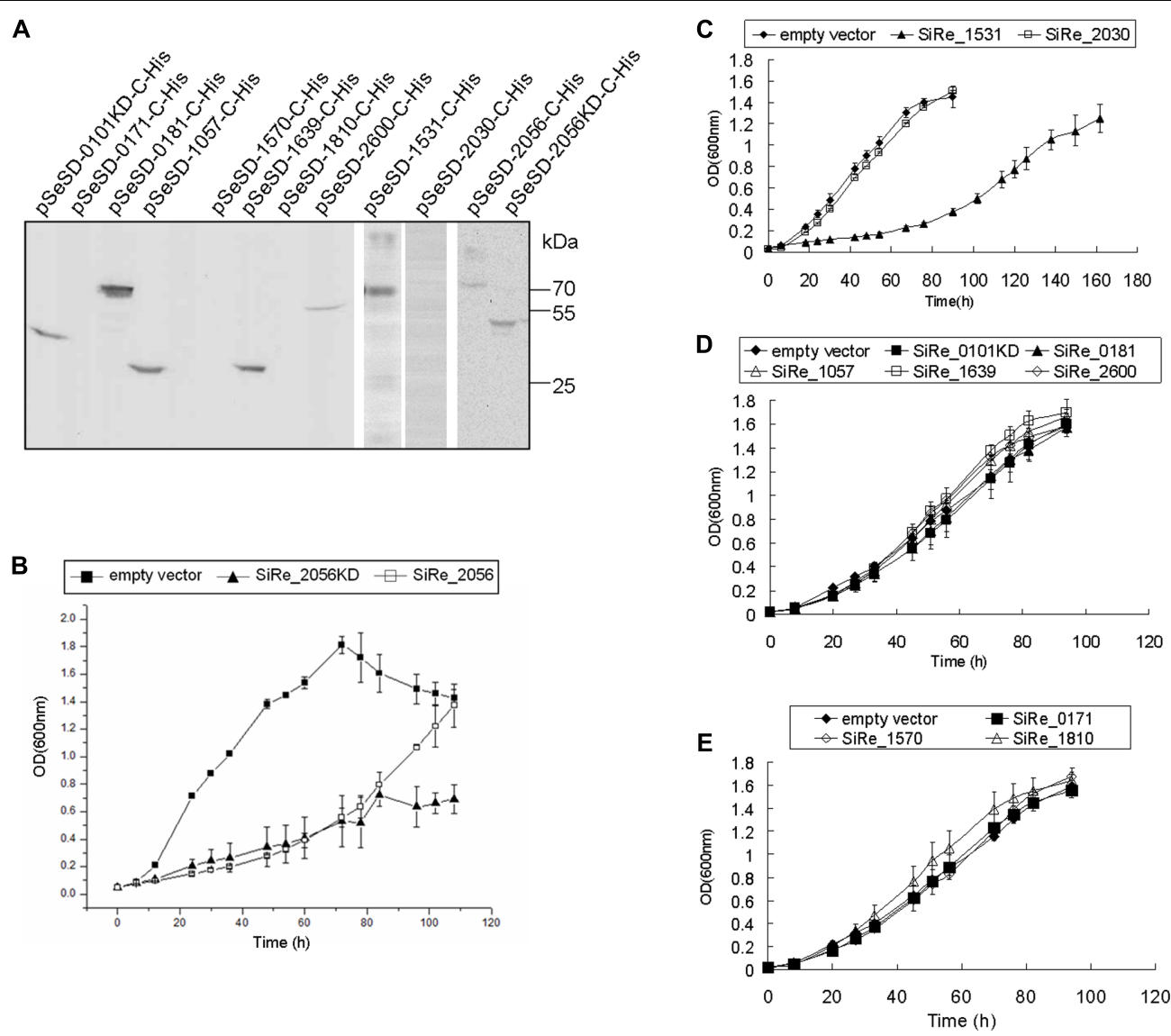

FIGURE 5 | The effects of ePKs overexpression on the growth of S. islandicus REY15A. (A) Western blot analysis of ePK (or ePK-KD) expressed in S. islandicus. The samples were taken from cultures at mid-log phase $(\mathrm{OD} \sim 0.4-0.6)$ for Western blot using anti-His-tag antibody. Protein size markers are indicated on the right. (B-E) Growth curves of each ePK (or ePK-KD) overexpression strain. The OD values were measured every 6 or $12 \mathrm{~h}$. The growth curves were obtained from three independent cultures. The bars indicate standard deviation.

Kisseleva-Romanova et al., 2006). In the Methanocaldococcus jannaschii genome, The Bud32 and Kae1 genes are fused into one ORF indicating they also interact with each other (Hecker et al., 2008). M. jannaschii and Pyrococcus abyssi Kae1 inhibited Bud32 autophosphorylation ability in vitro (Perrochia et al., 2013). The inactivation of Bud32 by Kael was also found in yeast (Hecker et al., 2008). The finding that overexpression of yeast Bud32 is toxic for the cell is similar to our result showing that SiRe_1570 could not be overexpressed in S. islandicus (Figure 5) (Hecker et al., 2008). Both our result of SiRe_1570 gene deletion using CRISPR-Cas system (data not shown) and that on S. acidocaldarius by markerless deletion or marker insertion (Hoffmann et al., 2017) showed that a SiRe_1570 deletion mutant could not be obtained, indicating that it might be an essential gene for cell viability. Collectively, SiRe_1570 probably has evolutionally conserved functions among archaea and eukaryotes.

SiRe_0171 (RIO1) and SiRe_1810 (RIO2) are two putative RIO protein kinases. The RIO kinase family is also ancient and found in all three domains of life. It was shown that eukaryotic RIOs participate in ribosome biogenesis, cell cycle progression, and genome integrity (Angermayr et al., 2002; Ferreira-Cerca et al., 2012; Widmann et al., 2012). H. volcanii RIO1 phosphorylated the $\alpha$ subunit of $20 \mathrm{~S}$ proteasome on Ser and Thr sites (Humbard et al., 2010), while S. solfataricus RIO1 was transcriptionally induced at early stage of UVtreatment (Gotz et al., 2007). The structure of Archaeoglobus fulgidus RIO2 has been resolved (LaRonde-LeBlanc et al., 2005) and P. horikoshii RIO2 was able to phosphorylate aIF2 $\alpha$ in vitro (Tahara et al., 2004). In $\gamma$-irradiation-treated P. furiosus, the mRNA level of RIO1 increased while that of RIO2 decreased (Williams et al., 2007). Our previous study showed that $\mathrm{SiRe}$-0171 was down-regulated in S. islandicus treated by DNA alkylating agents methyl methanesulfonate (MMS) (Song et al., 2016). Here, we found that both RIOs displayed autophosphorylation ability and could not be overexpressed in S. islandicus, suggesting that they activated themselves in important cellular pathways (cell cycle regulation or DNA damage response) and their expressions were strictly regulated in the cell.

The SiRe_0181 homolog in S. acidocaldarius is one of the pilus components, AapE, and may participate in virus infection 


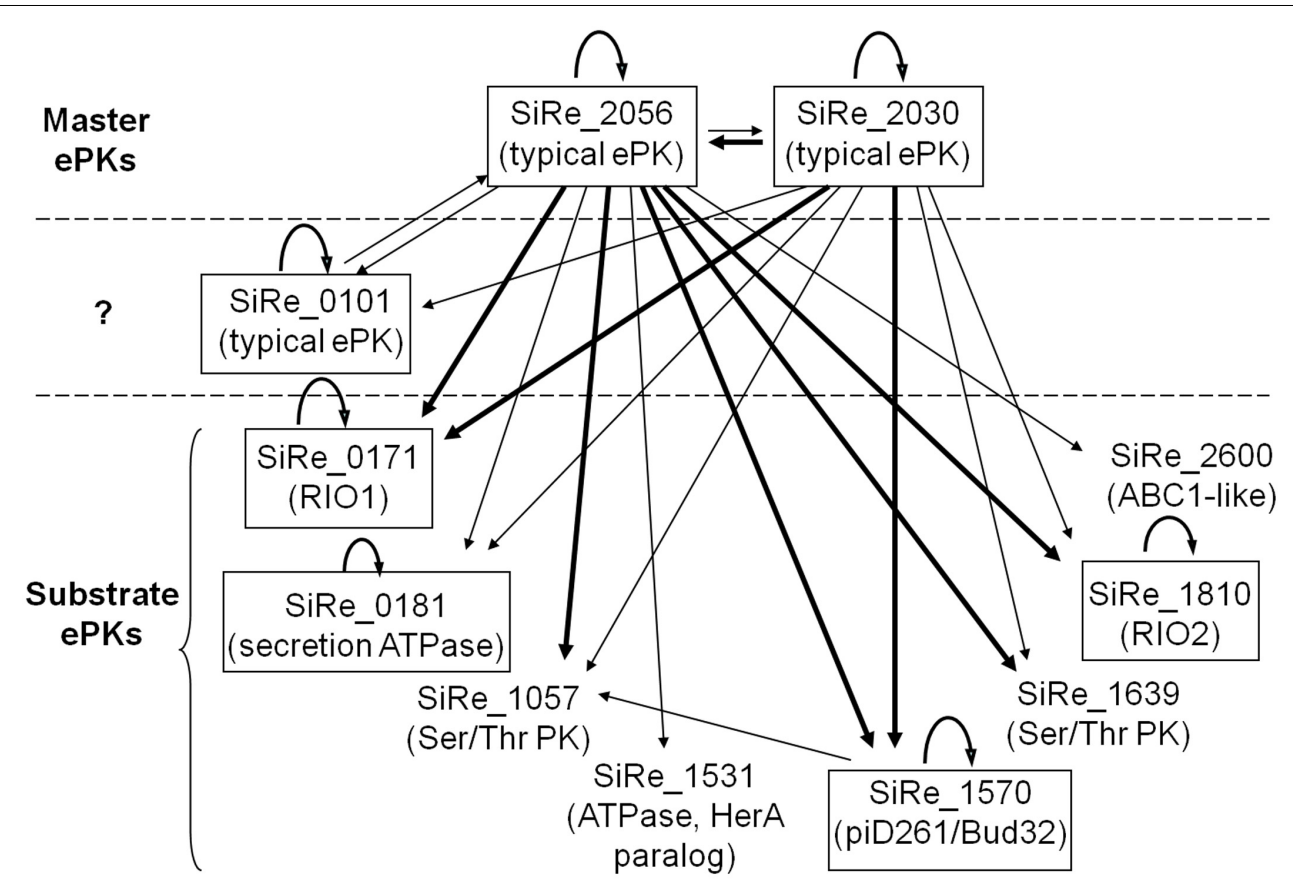

FIGURE 6 | A proposed regulatory network of S. islandicus ePKs according to auto- and cross-phosphorylation results. The two putative master regulator kinases are SiRe_2030 and SiRe_2056 which exhibit autophosphorylation activities and efficiently phosphorylate on most of the other ePKs. In addition, SiRe_0101 contains TM domain and phosphorylates SiRe_2056KD in vitro, suggesting that it may work as an accessory kinase for the master kinase that responds to an extracellular signal and spread the signal through SiRe_2056. On the other hand, the substrate kinases are those that do not transfer phosphates to the other ePKs. The ePKs having autophosphorylation activities are indicated in boxes. The arrow indicates the direction of phosphorylation, in which the thick lines represent high activities. The names of the most closely related protein kinase subfamily are shown in the parentheses (see the text for details).

and the expression of Sulfolobus pilus (Henche et al., 2012; Deng et al., 2014). It is annotated as a secretion ATPase and its homolog in S. solfataricus, SsoPK2, was the only one having autophosphorylation activity among five $S$. solfataricus secretion ATPase (Lower and Kennelly, 2003; Albers and Driessen, 2005). Our results revealed that the Walker A (and/or Walker B) motif, but not the potential HRD motif identified previously, is essential for the autophosphorylation activity of SiRe_0181, indicating a different mechanism for autophosphorylation.

We showed that SiRe_1531 could not phosphorylate itself and its overexpression inhibited cell growth. Bioinformatics analysis also revealed that it is a paralog of Helicase/ATPase HerA which is involved in DNA end resection of homologous recombination (Hopkins and Paull, 2008; Huang et al., 2015). HerA overexpression led to enlarged cells with multichromosomes and reduced viability, probably affecting normal DNA metabolism (unpublished data). Therefore, overexpression of SiRe_1531 might have similar effect on the cell by interfering with DNA metabolism, resulting in growth retardance.

For other ePKs which did not have autophosphorylation activity, SiRe_1057, SiRe_1639, and SiRe_2600, no previous investigation was reported. In NCBI, SiRe_2600 is annotated as $\mathrm{ABC} 1$ kinases that might be involved in ubiquinone biosynthesis, whereas SiRe_1057 and SiRe_1639 are only annotated as Ser/Thr
PKs. Our current results showed that all the ePKs were only phosphorylated by heterologous ePKs in vitro and each can be overexpressed in S. islandicus, indicating that they might locate at the bottom of the hierarchy network which needs to be activated by upstream ePKs.

Based on the phosphorylation of the ePKs on themselves and the other ePKs, we propose a putative regulatory network of $S$. islandicus ePKs (Figure 6). It is composed of two distinct functional classes: master regulator kinases and substrate kinases. SiRe_2030 and SiRe_2056 exhibited higher autophosphorylation activities and phosphorylation on other ePKs and therefore can be defined as the master regulator kinases. Since SiRe_0101 also contains a TM domain and phosphorylates SiRe_2056KD, it may serve as an accessory kinase for the master kinase sensing the extracellular signals. The base of the regulatory network is built up by the substrate kinases that were phosphorylated by master kinases and do not have phosphorylation activity on other ePKs. The fact in the network that one master ePK is able to phosphorylate a number of substrate ePKs while a substrate $\mathrm{ePK}$ can also be phosphorylated by several master ePKs (mainly by SiRe_2030 and SiRe_2056) is similar to those in eukaryotes and bacteria. However, only two regulatory layers exist in S. islandicus ePK network, suggesting that it is simpler than those in the other two domains, which have multiple-layered 
regulatory network. In addition, it seems that the typical ePKs, which emerged later than the ancient RIOs and Bud32 kinases, become the master kinases. We speculate that the RIOs and Bud32 are ancient and evolutionarily conserved kinases which should have conserved functions in vivo. While during a long time of evolution, species need new strategies for responding to new environments and survival. That could be the reason why the ePKs like SiRe_0101, SiRe_2056 and SiRe_2030 emerged, which contain a TM domain for membrane localization and sensing the extra-cellular signals and/or a TPR that mediate protein-protein interactions. Consistently, both master kinases in M. tuberculosis, PknB and PknH, contain TM and folded extracellular sensor domains (Baer et al., 2014). In addition, the low conservation of these ePKs in archaea (SiRe_0101 and SiRe_2056 even do not exist in several Sulfolobus species) is in agreement with our speculation that they emerged later to sense various signals in different environments. The autophosphorylation ability of the RIOs and Bud32 kinases also indicates that these kinases may not completely depend on the master kinases, but activate themselves in some essential conserved pathways.

\section{AUTHOR CONTRIBUTIONS}

$\mathrm{QH}$ designed the project, conducted most of the experiments, analyzed the data, and wrote draft of the paper. QZ and JM performed part of the experiments in plasmid construction and

\section{REFERENCES}

Albers, S. V., and Driessen, A. J. (2005). Analysis of ATPases of putative secretion operons in the thermoacidophilic archaeon Sulfolobus solfataricus. Microbiology 151(Pt 3), 763-773. doi: 10.1099/mic.0.27699-0

Angermayr, M., Roidl, A., and Bandlow, W. (2002). Yeast Riolp is the founding member of a novel subfamily of protein serine kinases involved in the control of cell cycle progression. Mol. Microbiol. 44, 309-324. doi: 10.1046/j.1365-2958. 2002.02881.x

Baer, C. E., Iavarone, A. T., Alber, T., and Sassetti, C. M. (2014). Biochemical and spatial coincidence in the provisional Ser/Thr protein kinase interaction network of Mycobacterium tuberculosis. J. Biol. Chem. 289, 20422-20433. doi: 10.1074/jbc.M114.559054

Boyer, J., Badis, G., Fairhead, C., Talla, E., Hantraye, F., Fabre, E., et al. (2004). Large-scale exploration of growth inhibition caused by overexpression of genomic fragments in Saccharomyces cerevisiae. Genome Biol. 5, R72. doi: 10.1186/gb-2004-5-9-r72

Breitkreutz, A., Choi, H., Sharom, J. R., Boucher, L., Neduva, V., Larsen, B., et al. (2010). A global protein kinase and phosphatase interaction network in yeast. Science 328, 1043-1046. doi: 10.1126/science.1176495

Casino, P., Rubio, V., and Marina, A. (2010). The mechanism of signal transduction by two-component systems. Curr. Opin. Struct. Biol. 20, 763-771. doi: 10.1016/ j.sbi.2010.09.010

Chu, H., and Wang, A. H. (2007). Enzyme-substrate interactions revealed by the crystal structures of the Archaeal Sulfolobus PTP-Fold phosphatase and its phosphopeptide complexes. Proteins 66, 996-1003. doi: 10.1002/prot.21262

Cohen, P. (2002). Protein kinases-the major drug targets of the twenty-first century? Nat. Rev. Drug Discov. 1, 309-315. doi: 10.1038/nrd773

Dardick, C., Schwessinger, B., and Ronald, P. (2012). Non-arginine-aspartate (non-RD) kinases are associated with innate immune receptors that recognize conserved microbial signatures. Curr. Opin. Plant Biol. 15, 358-366. doi: 10.1016/j.pbi.2012.05.002 protein purification. JM and JN helped revised the manuscript. YS conceived the idea for the project and helped write the paper. All authors approved the version to be published and agreed to be accountable for all aspects of the work in ensuring that questions related to the accuracy or integrity of any part of the work are appropriately investigated and resolved.

\section{FUNDING}

This work was supported by the National Natural Science Foundation of China (31470184 and 31670061 to YS) and China Postdoctoral Science Foundation (2016M602130 to QH).

\section{ACKNOWLEDGMENT}

We thank Mr. Junfeng Liu for providing SiRe_2030 overexpression strain and Ms. Xiaohui Zhao for providing SiRe_2056KD overexpression strain.

\section{SUPPLEMENTARY MATERIAL}

The Supplementary Material for this article can be found online at: https://www.frontiersin.org/articles/10.3389/fmicb. 2017.02173/full\#supplementary-material

Deng, L., He, F., Bhoobalan-Chitty, Y., Martinez-Alvarez, L., Guo, Y., and Peng, X. (2014). Unveiling cell surface and type IV secretion proteins responsible for archaeal rudivirus entry. J. Virol. 88, 10264-10268. doi: 10.1128/JVI.01495-14

Deng, L., Zhu, H., Chen, Z., Liang, Y. X., and She, Q. (2009). Unmarked gene deletion and host-vector system for the hyperthermophilic crenarchaeon Sulfolobus islandicus. Extremophiles 13, 735-746. doi: 10.1007/s00792-0090254-2

Donnelly, N., Gorman, A. M., Gupta, S., and Samali, A. (2013). The eIF2alpha kinases: their structures and functions. Cell Mol. Life Sci. 70, 3493-3511. doi: 10.1007/s00018-012-1252-6

Downey, M., Houlsworth, R., Maringele, L., Rollie, A., Brehme, M., Galicia, S., et al. (2006). A genome-wide screen identifies the evolutionarily conserved KEOPS complex as a telomere regulator. Cell 124, 1155-1168. doi: 10.1016/j.cell.2005. 12.044

Esser, D., Hoffmann, L., Pham, T. K., Brasen, C., Qiu, W., Wright, P. C., et al. (2016). Protein phosphorylation and its role in archaeal signal transduction. FEMS Microbiol. Rev. 40, 625-647. doi: 10.1093/femsre/fuw020

Esser, D., Pham, T. K., Reimann, J., Albers, S. V., Siebers, B., and Wright, P. C. (2012). Change of carbon source causes dramatic effects in the phospho-proteome of the archaeon Sulfolobus solfataricus. J. Proteome Res. 11, 4823-4833. doi: 10.1021/pr300190k

Ferreira-Cerca, S., Sagar, V., Schafer, T., Diop, M., Wesseling, A. M., Lu, H., et al. (2012). ATPase-dependent role of the atypical kinase Rio2 on the evolving pre40 S ribosomal subunit. Nat. Struct. Mol. Biol. 19, 1316-1323. doi: 10.1038/ nsmb. 2403

Frols, S., Gordon, P. M., Panlilio, M. A., Duggin, I. G., Bell, S. D., Sensen, C. W., et al. (2007). Response of the hyperthermophilic archaeon Sulfolobus solfataricus to UV damage. J. Bacteriol. 189, 8708-8718. doi: 10.1128/JB. 01016-07

Gotz, D., Paytubi, S., Munro, S., Lundgren, M., Bernander, R., and White, M. F. (2007). Responses of hyperthermophilic crenarchaea to UV irradiation. Genome Biol. 8:R220. doi: 10.1186/gb-2007-8-10-r220 
Groves, M. R., and Barford, D. (1999). Topological characteristics of helical repeat proteins. Curr. Opin. Struct. Biol. 9, 383-389. doi: 10.1016/S0959-440X(99) 80052-9

Guo, L., Brugger, K., Liu, C., Shah, S. A., Zheng, H., Zhu, Y., et al. (2011). Genome analyses of Icelandic strains of Sulfolobus islandicus, model organisms for genetic and virus-host interaction studies. J. Bacteriol. 193, 1672-1680. doi: 10.1128/JB.01487-10

Haile, J. D., and Kennelly, P. J. (2011). The activity of an ancient atypical protein kinase is stimulated by ADP-ribose in vitro. Arch. Biochem. Biophys. 511, 56-63. doi: $10.1016 /$ j.abb.2011.04.006

Hanks, S. K. (2003). Genomic analysis of the eukaryotic protein kinase superfamily: a perspective. Genome Biol. 4, 111. doi: 10.1186/gb-2003-4-5-111

Hanks, S. K., and Hunter, T. (1995). Protein kinases 6. The eukaryotic protein kinase superfamily: kinase (catalytic) domain structure and classification. FASEB J. 9, 576-596.

Haurat, M. F., Figueiredo, A. S., Hoffmann, L., Li, L., Herr, K., J Wilson, A., et al. (2017). ArnS, a kinase involved in starvation-induced archaellum expression. Mol. Microbiol. 103, 181-194. doi: 10.1111/mmi.13550

Hecker, A., Graille, M., Madec, E., Gadelle, D., Le Cam, E., van Tilbergh, H., et al. (2009). The universal Kael protein and the associated Bud32 kinase (PRPK), a mysterious protein couple probably essential for genome maintenance in Archaea and Eukarya. Biochem. Soc. Trans. 37(Pt 1), 29-35. doi: 10.1042/ BST0370029

Hecker, A., Lopreiato, R., Graille, M., Collinet, B., Forterre, P., Libri, D., et al. (2008). Structure of the archaeal Kael/Bud32 fusion protein MJ1130: a model for the eukaryotic EKC/KEOPS subcomplex. EMBO J. 27, 2340-2351. doi: 10.1038/emboj.2008.157

Henche, A. L., Ghosh, A., Yu, X., Jeske, T., Egelman, E., and Albers, S. V. (2012). Structure and function of the adhesive type IV pilus of Sulfolobus acidocaldarius. Environ. Microbiol. 14, 3188-3202. doi: 10.1111/j.1462-2920. 2012.02898.x

Hoffmann, L., Schummer, A., Reimann, J., Haurat, M. F., Wilson, A. J., Beeby, M., et al. (2017). Expanding the archaellum regulatory network - the eukaryotic protein kinases ArnC and ArnD influence motility of Sulfolobus acidocaldarius. Microbiologyopen 6:e0414. doi: 10.1002/mbo3.414

Hopkins, B. B., and Paull, T. T. (2008). The P. furiosus mre11/rad50 complex promotes $5^{\prime}$ strand resection at a DNA double-strand break. Cell 135, 250-260. doi: 10.1016/j.cell.2008.09.054

Huang, Q., Liu, L., Liu, J., Ni, J., She, Q., and Shen, Y. (2015). Efficient 5' $5^{\prime}$ DNA end resection by HerA and NurA is essential for cell viability in the crenarchaeon Sulfolobus islandicus. BMC Mol. Biol. 16:2. doi: 10.1186/s12867015-0030-Z

Humbard, M. A., Reuter, C. J., Zuobi-Hasona, K., Zhou, G., and Maupin-Furlow, J. A. (2010). Phosphorylation and methylation of proteasomal proteins of the haloarcheon Haloferax volcanii. Archaea 2010:481725. doi: 10.1155/2010/ 481725

Humphrey, S. J., James, D. E., and Mann, M. (2015). Protein phosphorylation: a major switch mechanism for metabolic regulation. Trends Endocrinol. Metab. 26, 676-687. doi: 10.1016/j.tem.2015.09.013

Johnson, L. N., Noble, M. E., and Owen, D. J. (1996). Active and inactive protein kinases: structural basis for regulation. Cell 85, 149-158. doi: 10.1016/S00928674(00)81092-2

Kennelly, P. J. (2003). Archaeal protein kinases and protein phosphatases: insights from genomics and biochemistry. Biochem. J. 370(Pt 2), 373-389. doi: 10.1042/ BJ20021547

Kennelly, P. J. (2014). Protein Ser/Thr/Tyr phosphorylation in the Archaea. J. Biol. Chem. 289, 9480-9487. doi: 10.1074/jbc.R113.529412

Kisseleva-Romanova, E., Lopreiato, R., Baudin-Baillieu, A., Rousselle, J. C., Ilan, L., Hofmann, K., et al. (2006). Yeast homolog of a cancer-testis antigen defines a new transcription complex. EMBO J. 25, 3576-3585. doi: 10.1038/sj.emboj. 7601235

Krupa, A., Preethi, G., and Srinivasan, N. (2004). Structural modes of stabilization of permissive phosphorylation sites in protein kinases: distinct strategies in Ser/Thr and Tyr kinases. J. Mol. Biol. 339, 1025-1039. doi: 10.1016/j.jmb.2004. 04.043

LaRonde-LeBlanc, N., Guszczynski, T., Copeland, T., and Wlodawer, A. (2005). Autophosphorylation of Archaeoglobus fulgidus Rio2 and crystal structures of its nucleotide-metal ion complexes. FEBS J. 272, 2800-2810. doi: 10.1111/j. 1742-4658.2005.04702.x

Lower, B. H., and Kennelly, P. J. (2003). Open reading frame sso2387 from the archaeon Sulfolobus solfataricus encodes a polypeptide with protein-serine kinase activity. J. Bacteriol. 185, 3436-3445. doi: 10.1128/JB.185.11.3436-3445. 2003

Lower, B. H., Potters, M. B., and Kennelly, P. J. (2004). A phosphoprotein from the archaeon Sulfolobus solfataricus with protein-serine/threonine kinase activity. J. Bacteriol. 186, 463-472. doi: 10.1128/JB.186.2.463-472.2004

Lundgren, M., and Bernander, R. (2007). Genome-wide transcription map of an archaeal cell cycle. Proc. Natl. Acad. Sci. U.S.A. 104, 2939-2944. doi: 10.1073/ pnas.0611333104

Mai, B., Frey, G., Swanson, R. V., Mathur, E. J., and Stetter, K. O. (1998). Molecular cloning and functional expression of a protein-serine/threonine phosphatase from the hyperthermophilic archaeon Pyrodictium abyssi TAG11. J. Bacteriol. 180, 4030-4035.

Makarova, K. S., Sorokin, A. V., Novichkov, P. S., Wolf, Y. I., and Koonin, E. V. (2007). Clusters of orthologous genes for 41 archaeal genomes and implications for evolutionary genomics of archaea. Biol. Direct 2:33. doi: 10.1186/17456150-2-33

Manning, G., Whyte, D. B., Martinez, R., Hunter, T., and Sudarsanam, S. (2002). The protein kinase complement of the human genome. Science 298, 1912-1934. doi: 10.1126/science.1075762

Nolen, B., Taylor, S., and Ghosh, G. (2004). Regulation of protein kinases; controlling activity through activation segment conformation. Mol. Cell 15, 661-675. doi: 10.1016/j.molcel.2004.08.024

Peng, N., Deng, L., Mei, Y., Jiang, D., Hu, Y., Awayez, M., et al. (2012). A synthetic arabinose-inducible promoter confers high levels of recombinant protein expression in hyperthermophilic archaeon Sulfolobus islandicus. Appl. Environ. Microbiol. 78, 5630-5637. doi: 10.1128/AEM.00855-12

Pereira, S. F., Goss, L., and Dworkin, J. (2011). Eukaryote-like serine/threonine kinases and phosphatases in bacteria. Microbiol. Mol. Biol. Rev. 75, 192-212. doi: 10.1128/MMBR.00042-10

Perrochia, L., Guetta, D., Hecker, A., Forterre, P., and Basta, T. (2013). Functional assignment of KEOPS/EKC complex subunits in the biosynthesis of the universal t6A tRNA modification. Nucleic Acids Res. 41, 9484-9499. doi: 10.1093/nar/gkt720

Podgornaia, A. I., and Laub, M. T. (2013). Determinants of specificity in two-component signal transduction. Curr. Opin. Microbiol. 16, 156-162. doi: 10.1016/j.mib.2013.01.004

Ray, W. K., Potters, M. B., Haile, J. D., and Kennelly, P. J. (2015). Activation of SsoPK4, an Archaeal eIF2alpha Kinase Homolog, by Oxidized CoA. Proteomes 3, 89-116. doi: 10.3390/proteomes3020089

Reimann, J., Esser, D., Orell, A., Amman, F., Pham, T. K., Noirel, J., et al. (2013). Archaeal signal transduction: impact of protein phosphatase deletions on cell size, motility, and energy metabolism in Sulfolobus acidocaldarius. Mol. Cell Proteomics 12, 3908-3923. doi: 10.1074/mcp.M113.027375

Reimann, J., Lassak, K., Khadouma, S., Ettema, T. J., Yang, N., Driessen, A. J., et al. (2012). Regulation of archaella expression by the FHA and von Willebrand domain-containing proteins ArnA and ArnB in Sulfolobus acidocaldarius. Mol. Microbiol. 86, 24-36. doi: 10.1111/j.1365-2958.2012.08186.x

Shi, L., Pigeonneau, N., Ravikumar, V., Dobrinic, P., Macek, B., Franjevic, D., et al. (2014). Cross-phosphorylation of bacterial serine/threonine and tyrosine protein kinases on key regulatory residues. Front. Microbiol. 5:495. doi: 10.3389/ fmicb.2014.00495

Shi, Y. (2009). Serine/threonine phosphatases: mechanism through structure. Cell 139, 468-484. doi: 10.1016/j.cell.2009.10.006

Solow, B., Young, J. C., and Kennelly, P. J. (1997). Gene cloning and expression and characterization of a toxin-sensitive protein phosphatase from the methanogenic archaeon Methanosarcina thermophila TM-1. J. Bacteriol. 179, 5072-5075. doi: 10.1128/jb.179.16.5072-5075.1997

Song, X., Huang, Q., Ni, J., Yu, Y., and Shen, Y. (2016). Knockout and functional analysis of two DExD/H-box family helicase genes in Sulfolobus islandicus REY15A. Extremophiles 20, 537-546. doi: 10.1007/s00792-016-0847-5

Spudich, J. L., and Stoeckenius, W. (1980). Light-regulated retinal-dependent reversible phosphorylation of Halobacterium proteins. J. Biol. Chem. 255, 5501-5503. 
Srinivasan, M., Mehta, P., Yu, Y., Prugar, E., Koonin, E. V., Karzai, A. W., et al. (2011). The highly conserved KEOPS/EKC complex is essential for a universal tRNA modification, t6A. EMBO J. 30, 873-881. doi: 10.1038/emboj.2010.343

Stevenson, L. F., Kennedy, B. K., and Harlow, E. (2001). A large-scale overexpression screen in Saccharomyces cerevisiae identifies previously uncharacterized cell cycle genes. Proc. Natl. Acad. Sci. U.S.A. 98, 3946-3951. doi: 10.1073/pnas.051013498

Tahara, M., Ohsawa, A., Saito, S., and Kimura, M. (2004). In vitro phosphorylation of initiation factor 2 alpha (aIF2 alpha) from hyperthermophilic archaeon Pyrococcus horikoshii OT3. J. Biochem. 135, 479-485. doi: 10.1093/jb/mvh055

Widmann, B., Wandrey, F., Badertscher, L., Wyler, E., Pfannstiel, J., Zemp, I., et al. (2012). The kinase activity of human Riol is required for final steps of cytoplasmic maturation of 40S subunits. Mol. Biol. Cell 23, 22-35. doi: 10.1091/ mbc.E11-07-0639
Williams, E., Lowe, T. M., Savas, J., and DiRuggiero, J. (2007). Microarray analysis of the hyperthermophilic archaeon Pyrococcus furiosus exposed to gamma irradiation. Extremophiles 11, 19-29. doi: 10.1007/s00792-006-0002-9

Conflict of Interest Statement: The authors declare that the research was conducted in the absence of any commercial or financial relationships that could be construed as a potential conflict of interest.

Copyright (c) 2017 Huang, Zhong, Mayaka, Ni and Shen. This is an open-access article distributed under the terms of the Creative Commons Attribution License (CC BY). The use, distribution or reproduction in other forums is permitted, provided the original author(s) or licensor are credited and that the original publication in this journal is cited, in accordance with accepted academic practice. No use, distribution or reproduction is permitted which does not comply with these terms. 